Hydroécol. Appl. (1992) Tome 4 Vol 1, pp. 21-36

\title{
Numerical modelling of tidal currents along the west European continental shelf
}

\author{
Jean-Marc Janin, Jean-Charles Galland \\ Laboratoire National d'Hydraulique (EDF) \\ 6. quai Watier - B.P. 49 - 78401 Chatou Cedex - France
}

\begin{abstract}
A tidal simulation, with a 2D code (TELEMAC) has been performed by the L.N.H. (Laboratoire National d'Hydraulique of Electricité de France) during a complete moon cycle in order to calculate current fields in the English Channel and the close Atlantique. These tidal currents combined with wind-induced-currents are aimed at predicting the movements of polluted matters. They will be used also as boundary conditions for modelling local areas.

The study area as it ends farther off the continental shelf encloses an exceptionnal number of elements $(20,000)$. However, the recent improvements in numerical methods and computers allow this calculation with a reasonable cost. The finite element technic is also used very favourably as the surface of an element decreases from 500 square miles along the west boundary to 1 square mile along the coast. Consequently, a new performance has been reached: modelling tidal currents inside a very large oceanic area with an extreme precision along the coast.
\end{abstract}

Key words. - English Channel, Bay of Biscay, Tides, Boundary conditions, Vertical profile of currents.

Résumé. - Une simulation de la marée a été réalisée au L.N.H. (Laboratoire National d'Hydraulique d'Électricité de France), à l'aide d'un code 2D (TELEMAC), pendant un cycle lunaire entier afin de calculer les champs de courants en Manche et dans le proche Atlantique. Ces courants de marée peuvent être utilisés pour générer les conditions aux limites de modèles locaux 3D. Combinés aux courants induits par le vent ils permettent de prédire le déplacement de matières polluantes.

La zone étudiée, en raison de sa grande extension au-delà du plateau continental, comporte un nombre exceptionnellement élevé d'éléments $(20000)$. Les récents progrès, portant à la fois sur les méthodes numériques et les calculateurs, rendent ces calculs possibles pour un coût raisonnable. La méthode des éléments finis s'avère bien adaptée à la représentation spatiale du domaine d'étude : l'aire d'un élément décroît ainsi de 500 miles carrés à la frontière occidentale du modèle jusqu'à 1 mile carré le long de la côte. En conséquence, il a été possible pour la première fois de modéliser les courants marins dans une très grande zone océanique avec une grande précision dans les régions côtières.

Mots clés. - Manche, Baie de Biscay, Marées, Conditions aux limites, Profil vertical de courants. 


\section{INTRODUCTION}

The most commonly encountered type of tide is that in which two high waters and two low waters occur each day, the times of high water and low water becoming later by approximately 50 minutes from one day to the next. This type of tide is found around nearly all shores bordering on the Atlantic Ocean. It is dominated by the lunar semi-diurnal constituent, denoted by M2, having a period of $12 \mathrm{~h} 25$, which is half of a lunar day. It is also well known that the range of the tide, defined as the difference in height between a high water and the following low water, varies periodically with the phases of the moon. Tides of maximum range, known as spring tides, occur within a day or two of a new or a full moon. This sequence arises from the solar semi-diurnal constituent denoted by $\mathrm{S} 2$ and having a period of half a solar day, alternately reinforcing and opposing the lunar semidiurnal constituent. Both constituents described above, and also a third one called N2 due to the elliptic trajectory of the moon and which effect is similar to S2 but weaker, are taken into account in the L.N.H model.

Horizontal movements of water, the tidal currents, are totally associated with the vertical rise and fall of the sea surface and are very sensitive to variations of the range of the tide. They are represented by the same harmonic constituents as elevation but not necessarily with the same phases. The phase relationship between elevation and current varies from place to place and is dependant on whether the tidal wave behaves rather like a progressive wave or rather like a stationnary wave. Thanks to the numerical code TELEMAC, the L.N.H model. estimates all these parameters all over the domain regarding an average current : the depth-mean current. However, variation of tidal currents with depth are evaluated afterwards.

\section{DESCRIPTION OF THE NUMERICAL CODE TELEMAC}

The code TELEMAC [2] solves the bidimensional shallow-water equations with a finite element method. We assume that the pressure is hydrostatic and the velocities uniform throughout the vertical. We estimate afterwards the variation of tidal current with depth. A celerity-velocity formulation of the continuity and momentum equations is employed, written in the Mercator plan [3], including a diffusion term and two source terms, the bottom friction and the Coriolis force :

$$
\left\{\begin{array}{l}
2 c \frac{\partial c}{\partial t}+2 c \bar{u} \cdot \frac{1}{\cos \lambda} \operatorname{grad} c+\frac{c^{2}}{\cos \lambda} \operatorname{div} \bar{u}-\frac{c^{2}}{R} \operatorname{tg} \lambda \cdot \bar{v}=0 \\
\frac{\partial \bar{u}}{\partial t}+\left(\frac{\bar{u} \cdot \operatorname{grad}}{\cos \lambda}\right) \bar{u}+2 c \frac{1}{\cos \lambda} \operatorname{grad} c=\frac{1}{\cos ^{2} \lambda} \operatorname{div}(v \operatorname{grad} \bar{u})-g \frac{1}{\cos \lambda} \operatorname{grad} Z_{f}+\vec{S}
\end{array}\right.
$$


$\overline{\mathrm{u}}, \overline{\mathrm{v}}$ : components of the depth-mean current. $\bar{u}$

c : velocity of propagation (celerity)

$v$ : coefficient of horizontal eddy viscosity.

$\mathrm{Z}_{\mathrm{f}}$ : bottom level

\subsection{Source terms}

The bottom stress:

$$
\overrightarrow{S_{f}}=-\frac{g}{h C_{2}^{2}\|\bar{u}\| \bar{u}}
$$

$\mathrm{C}_{z}$ : Chézy coefficient.

$\mathrm{h}:$ depth of water.

The Coriolis force :

$$
\overrightarrow{S_{c}}=-2 \omega \text { T } \sin \lambda \vec{k} \wedge \bar{u}
$$

$\mathrm{w}_{\mathrm{T}}$ : angular rate ot rotation of the earth.

$\vec{h}$ : depth of water : vertical unitary vector.

\subsection{The algorithm}

The numerical method solves the equations by means of a decomposition in fractionals steps. Each numerical operator can this way be treated by an adequat method. The resolution is achieved in two steps: an advection step and a diffusion-free surfacecontinuity-pressure step.

The advection is computed through a characteristic curve method which is unconditionally stable. Both, the celerity and the velocity can be advected. However, in this tidal model, the current number is always lower than
$\overrightarrow{\mathrm{S}}$ : source terms.

g: gravity.

$\delta$ : latitude.

0.2 and the advection of the velocities is quite negligeable. The advection of the variable " $c$ " induces in our model a lack of conservativity especially along the shelf edge where the gradient of "c" is important. This effect is due to the interpolation of this variable at the foot of the characteristic. It has been greatly reduced by refining the mesh around the edge.

Then, diffusion terms, source terms and free surface-continuity terms are taken into account. The adopted space discretization is a finite element discretization in triangles. A temporal discretization is performed before so that non linearities are removed. The obtained linear system is finally solved by iterative operations of the conjugate gradient method.

\section{STUDY DOMAIN AND BOUNDARY CONDITIONS}

\subsection{The choice of the domain}

An international working group [5] was formed in 1984 to assemble and disseminate a data set for tidal model testing. This group selected an area that encloses the English Channel and the meridional part of the North 
Sea. They supplied along the open boundaries a complete data field regarding amplitude and phase of elevation of the main tidal waves. Consequently, we have fixed our northern boundary in order to coincide with the one they had chosen.

However, as we wanted also to model at least the Atlantic coast of France, we needed a complete data field in the Bay of Biscay too. Unfortunately the amount of data is very small there and obliged us to move this boundary far away from the coast to damp the effect of the poor quality of the boundary conditions we could fix there. Besides, it should be noted that, in the Bay of Biscay, the shelf width ranges between more than 500 $\mathrm{km}$ in its northern part to almost $0 \mathrm{~km}$ close to the Spain border. It means that, if the west boundary is not sent far enough it will necesseraly cross the shelf edge, a weak spot of the model. In order to avoid that, we finally decided to fix this boundary along the 12.5 West meridian where the height of water is about $4,500 \mathrm{~m}$. We crossconnected it to the coast, in the south along the parallel of Cape Finistere (Spain), in the north along the parallel of Mizen Head (Ireland). The depth of water is about $4,500 \mathrm{~m}$ throughout the first link as there is no shelf there. The second link is crossed by the edge in its middle but it induces no effect as the main tidal waves, which propagate from South to North, are coming out the model there.

To enclose the shelf edge inside the model is favourable concerning the problem of the quality of the boundary conditions. As a matter of fact, the edge acts like a filter for the incoming waves: they are both reflected and diffracted by the steep bottom gradients, and the results of calculation near the coast are less dependent on the boundary conditions.

Two other open boundaries appear in the model. One is crossing the Irish Sea from Carnsore Point (Ireland) to Cape St David (Wales), the other one the Severn estuary. Both are fairly well documented [9].

The whole domain is shown on figure 3 .

\subsection{The tested boundary conditions}

Three kinds of boundary conditions have been successively tested; we called them:

- incident wave

- total wave tion

- specified elevation with correc-

\subsubsection{Incident wave}

The so called boundary condition has been tested first. It prescribes some relationship between elevation and current at the boundary such as a radiation condition. It takes the following form for a tidal wave with an angular frequency $w$ in a point $M$ at the time $t$ :

$$
c \vec{u} \cdot \vec{n}=g S-g(1-\vec{k} \cdot \vec{n}) \hat{S}
$$

$\vec{n}$ : unitary vector outgoing normally to the boundary in $\mathrm{M}$. 
$S$ : free surface elevation at the previous time step in $M$.

$\vec{k}$ : unitary vector in the direction of propagation of incident wave in $M$. $S=A(M) \cos (\omega t-\varphi(M))$ : sinusoïdal signal of incident wave elevation.

$A(M)$ and $j(M)$, respectively the amplitude and the phase of the incident wave in $M$, have to be estimated, which is the deficiency of this approach. As a matter of fact, the measures give the amplitude and the phase of the total wave and it leads to unacceptable phase shifts to consider that incident and total waves are equal even in deep water.

Therefore, we developped an iterative calculation with feedback in order to evaluate $A(M)$ and $\varphi(M)$ at every open boundary nodes. However, we got undesirable oscillations along the boundaries that we could not remove. This calculation reduced partially the shifts but we still had an important phase shift of $-23^{\circ}$ in $M 2$ elevation in the middle of the English Channel.

Note that this approach developped by the L.N.H. gave excellent results for several schematic test cases without any reflection along the open boundaries. Finally, this approach seems to be very interesting as long as we can get data about the incident wave.

\subsubsection{Total wave}

Another radiation condition formulated by Flather [7] has been used and quite successfully tested in this model. It is given for a wave with an angular frequency $\omega$ by :

$$
c \vec{u} \cdot \vec{n}=c \hat{u}+g(S-\hat{S})
$$

$\hat{u}=U(M) \operatorname{cs}(\omega t+\phi(M))$ : sinusoïdal signal homogeneous to a velocity. $\hat{A}=A(M) \cos (\omega t-\varphi(M))$ : sinusoïdal signal of total wave elevation.

On the contrary to the previous approach, this one supposes that $\hat{S}$ is the signal of the total wave elevation which is given by the measurements. However, it is necessary to define a new signal obtained by Fourier analysis of $\overrightarrow{\mathrm{u}} \cdot \overrightarrow{\mathrm{n}}(\mathrm{t})$. We achieved this calculation thanks to iterations with a duration multiple of the period of the considered wave.

We tested first this boundary condition in the main tidal wave M2. The iterative process presented before converged after 8 iterations of 4 periods of M2 wave each. Then we obtain amplitudes and phases regarding elevation of $\mathrm{M} 2$ in very good agreement with the measurements. The generated non linear wave M4 was also in reasonable agreement except in Saint-Malo gulf where it was slightly increased.

This formulation used for a single wave can be applied also for $\mathrm{n}$ waves of different angular frequencies $\omega_{\mathrm{i}}$ as it follows :

$$
c \vec{u} \cdot \vec{n}=c \hat{u}_{\text {tot }}+g\left(S-\hat{S}_{\text {tot }}\right)
$$

with

$\hat{\mathrm{u}}_{\mathrm{tot}}=\sum_{\mathrm{i}=1}^{\mathrm{n}} \hat{\mathrm{u}}_{\mathrm{i}}$ and $\hat{\mathrm{S}}_{\mathrm{tot}}=\sum_{\mathrm{i}=1}^{\mathrm{n}} \hat{\mathrm{S}}_{\mathrm{i}}$

However, in order to calculate at the end of each iteration with a Fourier 
analysis of $(t)$, it is necessary for the iteration duration to be long enough to seperate the waves.

The minimal duration is about :

$$
\frac{2 \pi}{\min \left|\omega_{i}-\omega_{j}\right|_{i \neq j}}
$$

It means that for the two main waves on the west European shelf, $\mathrm{M} 2$ and S2, 15 days of simulations are necessary for each iteration. If we suppose that we still need 8 iterations for the convergence, 4 months of simulation are expected to complete the calculation, that is to say several hours of CPU time of a Cray computer. Consequently, we decided to look for a third formulation of the boudary condition especially for the model calibration with several tidal waves.

\subsubsection{Specified elevation with cor- rection}

To specify elevation on open boundaries for a scheme without correction yields an unsteady calculation around the shelf edge after a couple of tidal cycles. As we said before this is a critical point of the model especially because of the advection of the velocity of propagation.

A recommended solution (see [1]) is to build a scheme that achieves each time step twice. As a matter of fact, at the end of the first lap we modify the advector current field used for the advection of the velocity of propagation in the second lap in order to centre it on time, whereas for the first lap we utilize the current field obtained at the previous time step as we used to do for a scheme without correction.

The effect of this correction is extremely positive : we get for the wave M2, after 4 tidal cycles, a result as good as the one we got with the radiation condition formulated by Flather. We decided finally to consider this approach for the whole study.

Another point is worth to be emphasised. As we split the resolution into two steps, with only one lap there is no influence of the second one to the first one. With the correction this influence exists. Usually such interdependence is positive to the stability and consequently the quality of a numerical model.

\section{SIMULATED COMPONENTS}

We decided to simulate and analyse with an adapted harmonic method a few waves all together. We wanted also to limit the duration of simulation to about one month. The harmonic method that we chose, adapted for short simulations, consists in minimizing the root-mean-square deviation. We applied it to the following 4 waves:

- M2 : principal lunar, semi-diurnal, period $44714 \mathrm{~s}$ (12h 25' 14")

- S2 : principal solar, semi-diurnal, period $43200 \mathrm{~s}$ (12h)

- N2: larger lunar elliptic, semidiumal, period $45570 \mathrm{~s}$ (12h 39' 30') 
- M4 : first harmonic of M2, quarter-diurnal, period $22357 \mathrm{~s}$ (6h 12' $37 ")$

The last wave is in fact specified only on shallow water open boundaries like the one in the middle of the North Sea. However, M4 is mostly generated inside the domain thanks to non linear effects applied to M2, such as the bottom stress.

The choice of these 4 waves can be explained as follows:

The first 3 ones are the strongest ones all over the English Channel and the close Atlantic Ocean. The average semi-amplitude of these waves obtained from ten offshore stations along the west European continental shelf are respectively 220,75 and $40 \mathrm{~cm}$.

The fourth wave in descending order is K2 with an average semi-amplitude of $22 \mathrm{~cm}$. Unfortunately, this wave which effect is to amplify the tidal range during equinoctial periods cannot be separated from S2 unless a simulation of 6 month is achieved. As a matter of fact, S2 and K2 periods (43 $082 \mathrm{~s}$ ) are very close to one another. It must be added that the behaviour of this two waves is quite similar and $\mathrm{K} 2$ components may be roughly deduced from S2.

The fifth wave is M4 with an average semi-amplitude of $18 \mathrm{~cm}$. Three reasons justify the choice we made in considering it in the harmonic analysis :

First, the average amplitude of this wave equals almost K2 but, as their spatial distributions are totally different, there exist many areas where
M4 is much more important than K2 (like Seine and Somme Bays or around Wight Island).

Also, M4 plays a prominent part in the asymmetry of the tide. One of its most remarkable effects is to create a deviation between the duration of the rising level and the duration of the falling level. Therefore, the amplitudes of the associated flood and ebb currents can slightly differ too. It seems interesting to reproduce this asymmetry whenever we are engaged in following polluted matters.

Last but not least, M4 is a test wave for the code because as we said before this wave is mainly produced inside the domain by the non linear terms of the shallow water equations. Now, usually the treatment of these terms is critical for numerical codes. TELEMAC, which is a fully non linear code produces a wave M4 really in agreement with the mesures.

\section{VARIATION OF TIDAL CURRENT WITH DEPTH}

From the depth mean-current computed by TELEMAC code, an evaluation of the current in the three-dimensional domain has been achieved subject to a few hypothesis (see [12]). Such a kind of calculation is judicious since the fluctuation of the tidal current along the vertical is low as compared with the depth-mean current. If we neglect density variations and suppose that the wind is weak, the fluctuation of current with depth is due entirely 
to the frictionnal stresses arising from bottom friction.

With this three-dimensional current field, it will be possible to appreciate more precisely the movements of polluted matters since their immersion depth is known. The vertical profiles of the current will be used also favourably as boundary conditions for local three-dimensional models and lastly, the estimation of the current at the surface will allow more realistic comparisons with the measures given by the Hydrographic Service (SHOM) [14].

\subsection{Hypothesis and equations}

Subsequently, we take into account the current generated by a single tidal wave with an angular frequency $w$. In comparison with what is commonly made in studying turbulent flow, we divide the current into a depth-mean current and a fluctuation as following :

$\overrightarrow{\mathrm{u}}=\overline{\mathrm{u}}+\overrightarrow{\mathrm{u}}$ with $\int_{-\mathrm{H}}^{0} \overrightarrow{\mathrm{u}^{\prime}} \mathrm{dz}=0$

The momentum equations in the $x$ and $y$ directions at any point $x, y, z$ in the water are written to $\vec{u}$ with the following hypothesis :

- advection terms are neglected for $\overrightarrow{\mathrm{u}}$ like for the depth-mean current $\overline{\mathrm{u}}$.

- horizontal viscosity of the fluctuation is neglected.

- we suppose that the coefficient of vertical eddy viscosity is independant of both $z$ and $t$. This hypothesis is not valid inside the bottom boundary layer that accounts for 10 to $20 \%$ of the depth of water.

- in order to be consistent with the harmonic analysis achieved on the depth-mean current at the considered angular frequency, we assume that the associated $\overrightarrow{\mathrm{u}}$ is a sinusoïdal signal with the same frequency. Therefore, just for the needs of this calculation, we use in the equation written to $\overline{\mathrm{u}}$ a partial bottom stress as follows :

$$
\overrightarrow{S_{c}}=-\frac{g U}{H_{C}^{2}} \bar{u}
$$

$U$ the average amplitude during a tidal period (12h25) of the entire depthmean current.

$\mathrm{H}$ mean water depth $\left(=-Z_{f}\right)$

Then, by substracting to the equations written to $\vec{u}$, the corresponding ones written to üand born of shallow water equations, we obtain :

$\left\{\begin{array}{l}\frac{\partial u^{\prime}}{\partial t}-f v^{\prime}-v_{z} \frac{\partial^{2} u^{\prime}}{\partial z^{2}}=\frac{g U}{H C_{z}^{2}} \bar{u} \\ \frac{\partial v^{\prime}}{\partial t}+f u^{\prime}-v_{z} \frac{\partial^{2} v^{\prime}}{\partial z^{2}}=\frac{g U}{H C_{z}^{2}} \bar{v}\end{array}\right.$

$v_{z}$ : coefficient of vertical eddy viscosity

$f$ : Coriolis parameter.

These are Ekman equations for a non permanent flow.

The previous system can be reduced into one equation by introducing the complex variables:

$Z=u^{\prime}+i v^{\prime}$ and $\bar{Z}=\bar{u}+i \bar{v}$

By defining the depth-mean components as :

$$
\bar{u}=\bar{u}_{x} \cos \omega t+\bar{u}_{y} \sin \omega t
$$


and $\quad \bar{v}=\bar{v}_{x} \cos \omega t+\bar{v}_{y} \sin \omega t$

we get :

$$
\bar{Z}=\bar{Z}_{1} \exp (i \omega t)+\bar{Z}_{2} \exp (-i \omega t)
$$

with :

$$
\left.\bar{z}_{1}=\frac{1}{2} \bar{u}_{x}-i \bar{u}_{y}+i \bar{v}_{x}+\bar{v}_{y}\right)
$$

and $\bar{Z}_{2}=\frac{1}{2}\left(\bar{u}_{x}+i \bar{u}_{y}+i \bar{v}_{x}-\bar{v}_{y}\right)$

Finally, the Ekman equation becomes :

$$
\begin{aligned}
& \frac{\partial Z}{\partial t}+i f Z-v_{z} \frac{\partial^{2} Z}{\partial z^{2}} \\
& =\frac{g U}{H C_{2}^{2}}\left[\bar{Z}_{1} \exp (i \omega t)+\bar{Z}_{2} \exp (-i \omega t)\right]
\end{aligned}
$$

\subsection{Resolution}

In order to solve this differential equation we take into account the following remarks.

- we neglect the effect of the wind on the surface and assume that stress there is null. It means that:

$$
\forall \mathrm{t}, \frac{\partial \mathrm{Z}}{\partial \mathrm{z}}(0)=0
$$

- we recall the definition of (8), the integration of which from the bottom to the surface equals zero:

$$
\forall \mathrm{t}, \int_{-\mathrm{H}}^{0} \mathrm{Z} d \mathrm{z}=0
$$

Let us now introduce a characteristic coefficient of vertical eddy viscosity no, and a joint dimensionless variable $K$ :

$v_{0}=\frac{g}{C_{z}^{2}} H U$ and $K=\frac{v_{0}}{v_{z}}$
Let us also introduce the dimensionless variables:

$$
\begin{aligned}
& \alpha_{H}=H(1+i) \sqrt{\frac{\omega+f}{2 v_{z}}} ; \\
& \beta_{H}=H(1-i) \sqrt{\frac{\omega-f}{2 v_{z}}} ; z^{*}=\frac{-z}{H}
\end{aligned}
$$

(note that $w-f$ is positive since we are dealing with waves, the periods of which are lower or equal than the period of a semi-diurnal wave).

In that case, we obtain :

$$
\begin{aligned}
Z & =\frac{K}{\alpha_{H}^{2}}\left\{1-\frac{\alpha_{H}}{S h \alpha_{H}} \operatorname{Ch}\left(\alpha_{H} Z^{*}\right)\right\} \bar{Z} \exp (i \omega t) \\
& +\frac{K}{\beta_{H}^{2}}\left\{1-\frac{\beta_{H}}{\operatorname{Sh} \beta_{H}} \operatorname{Ch}\left(\beta_{H} Z^{*}\right)\right\} \bar{Z}_{2} \exp (-i \omega t)
\end{aligned}
$$

The variables $\alpha_{H}$ and $\beta_{H}$ are directly connected to both Ekman number $\left(E=f^{2} / v_{z}\right)$ and Rossby number $(R=\omega / f)$ :

$$
\alpha_{H}^{2}=i(R+1) E
$$

and $\beta_{H^{2}}=-i(R-1) E$

We finally define two modified Ekman numbers, $E_{1}$ and $E_{2}$, as follows :

$$
\begin{aligned}
& \alpha_{H}=(1+i) E_{1} \\
& \beta_{H}=(1-i) E_{2}
\end{aligned} \text { and } \begin{aligned}
& 2 E_{T}^{2}=(R+1) E \\
& 2 E_{2}^{2}=(R-1) E
\end{aligned}
$$

Therefore :

$$
\begin{aligned}
Z & =f\left(E_{1}, 1\right) \bar{Z}_{1} \exp (i \omega t) \\
& +f\left(E_{2},-1\right) \bar{Z}_{2} \exp (-i \omega t)
\end{aligned}
$$

with :

$$
\begin{aligned}
& f\left(E_{k}, \varepsilon\right)=\frac{K}{2 \varepsilon i E_{k}^{2}} \\
& \quad\left\{1-\frac{(1+\varepsilon i) E_{k}}{\operatorname{Sh}\left[(1+\varepsilon i) E_{k}\right]} \text { Ch }\left[(1+\varepsilon i) E_{k} z^{*}\right]\right\}
\end{aligned}
$$




\subsection{Solution analysis}

In this paragraph, let us have a look at the solution in two special cases; a complete analysis would be too long and is not the purpose of this paper. The first case will deal with very shallow water, the second one with the current at the surface.

\subsubsection{If $E_{1}<0.7$}

In that case, as the modulus of $\mathrm{aH}_{\mathrm{H}}$ and $b_{H}$ are lower than 1 , we can substitute the hyperbolic terms in $Z$ for their Taylor expansions at the third order. The expression of $Z$ is mainly simplify to :

$$
Z=\frac{K}{6}\left(1-3 z^{* 2}\right) \bar{Z}
$$

Bowden, from mesurements, established the following formula valid outside the boundary layer that, according to him, accounts for $14.5 \%$ of the depth of water:

$$
\begin{aligned}
\overrightarrow{\mathrm{u}} & =\overrightarrow{\mathrm{u}}(0)\left(1-0.37 \frac{\mathrm{z}^{2}}{\mathrm{H}^{2}}\right) \\
& =1.14 \overline{\mathrm{u}}\left(1-0.37 \frac{\mathrm{z}^{2}}{\mathrm{H}^{2}}\right) \\
& =\bar{u}+0.14 \overline{\mathrm{u}}\left(1-3 \frac{\mathrm{z}^{2}}{\mathrm{H}^{2}}\right)
\end{aligned}
$$

Davies [10] suggested, outside the boundary layer, 2 formulations of the eddy viscosity :

$v_{\mathrm{z}}=0.0025 \mathrm{HU}$ so: $\mathrm{v}_{\mathrm{z}} \approx v_{0}$

and

$v_{z}=2.010^{-5} \frac{U^{2}}{f} \approx 0.2 U^{2}$

The first formulation which is rather valid for shallow water fits perfectly our expression to the mesures as it gives to $K$ the value 1 . The interest of the calculation is that it fixes the limits of validity of the law. If we introduce the parameter $\tau=\mathrm{H} / \mathrm{U}$, and choose for $\mathrm{C}_{\mathrm{z}}{ }^{2}$ the value $4000 \mathrm{~m} / \mathrm{s}^{2}$, we obtain that the law is valid until: $\tau<10$ s.

(Note that this law which is valid near the coast, set to be colinear along the profile)

\subsubsection{If $z^{*}=0$}

As the current at the free surface is the one usually measured, we have a special interest in evaluating it in order to achieve reliable comparisons. Furthermore, this current induces the movements of a large family of polluted matters: the ones lighter than sea surface water (oil products in particular).

For low values of Ekman number, as it has been established previously, the current at the surface generated by a tidal wave is nearly $15 \%$ greater than the mean-depth current and colinear to it. Further, a global study of $f\left(E_{k}, \varepsilon\right)$ is impossible. We resolve it into two functions, $a\left(E_{k}, \varepsilon\right)$ and b $\left(E_{k}, \varepsilon\right)$, respectively the real part and the imaginery part of $f\left(E_{k}, \varepsilon\right)$. Consequently, we get for $z^{*}=0$ :

$\mathrm{a}\left(E_{k}, \varepsilon\right)=K \frac{\operatorname{Ch} E_{k} \sin E_{k}-\operatorname{Sh} E_{k} \cos E_{k}}{E_{k}\left[\operatorname{Ch}\left(2 E_{k}\right)-\cos \left(2 E_{k}\right)\right]}$

$b\left(E_{k}, \varepsilon\right)=$

$\varepsilon K\left\{\frac{C h E_{k} \sin E_{k}+S h E_{k} \cos E_{k}}{E_{k}\left[\operatorname{Ch}\left(2 E_{k}\right)-\cos \left(2 E_{k}\right)\right]}-\frac{1}{2 E_{k}^{2}}\right\}$ 
As well as we did for (13), we can express as follows:

$$
u^{\prime}=u_{x}^{\prime} \cos \omega t+u_{y}^{\prime} \sin \omega t
$$

and

$$
v^{\prime}=v_{x}^{\prime} \cos \omega t+v_{y}^{\prime} \sin \omega t
$$

We finally obtain the following matrix that transforms the harmonic components of into the ones of $\overrightarrow{\mathrm{u}}$ :

$$
\left(\begin{array}{c}
u_{x}^{\prime} \\
u_{y}^{\prime} \\
v_{x}^{\prime} \\
v_{y}^{\prime}
\end{array}\right)=\left(\begin{array}{cccc}
a^{+} & -b^{-} & -b^{+} & -a^{-} \\
b^{-} & a^{+-} & a^{-} & -b^{+} \\
b^{+} & a^{-} & a^{+} & -b^{-} \\
-a^{-} & b^{+} & b^{-} & a^{+}
\end{array}\right)\left(\begin{array}{c}
\bar{u}_{x} \\
\bar{u}_{y} \\
\bar{v}_{x} \\
\bar{v}_{y}
\end{array}\right)
$$

with :

$$
\begin{aligned}
& a^{+}=\frac{a\left(E_{2},-1\right)+a\left(E_{1},+1\right)}{2} \\
& a^{-}=\frac{a\left(E_{2},-1\right)+a\left(E_{1},+1\right)}{2}
\end{aligned}
$$

and

$$
\begin{aligned}
& b^{+}=\frac{b\left(E_{2},-1\right)+b\left(E_{1},+1\right)}{2} \\
& b^{-}=\frac{b\left(E_{2},-1\right)+b\left(E_{1},+1\right)}{2}
\end{aligned}
$$

In order to calculate $\mathrm{a}^{+}, \mathrm{a}^{-}, \mathrm{b}^{+}$and $b^{-}$, we had also to estimate either $v_{z}$ or $\mathrm{K}$.

Both formula suggested by Davies [10] have their own validity range. The first one adjusts very well to shallow water as it equilibrates eddy viscosity and bottom stress. However, when the water depth is getting greater, this formula increases exaggeratedly the value of $v_{z}$ and the second one fits better. For $\tau$ equal to 80 seconds that we call latter $\tau_{0}$, the two formulae give the same result. Therefore, whenever $\tau$ is lower than $\tau_{0}$, we will try to be close to the first formulation, whenever $\tau$ is greater than $\tau_{0}$, we will try to be close to the second one.

If we consider $K$ we notice that:

- according to formula (27) : $K=1$

- according to formula (28) : $\mathrm{K}=\frac{\tau}{\tau_{0}}$

It exists a simple expression for $\mathrm{K}$ that tends quickly to the previous ones when $\tau$ deviates from $\tau_{0}$ and also avoids any kind of discontinuity in $\tau_{0}$. This expression is :

$$
\mathrm{K}=\operatorname{Coth} \frac{\tau_{0}}{\tau}
$$

Then, we obtain the values of the two modified Ekman numbers, $E_{1}$ and $E_{2}$, and consequently the ones of the matrix components (see figure 1) because $E_{1}$ and $E_{2}$ are related to the Ekman number (22), which is given by :

$$
E=K \frac{f H^{2}}{v_{0}} \approx K \frac{\tau}{25}
$$

On figure 2 one can see two comparisons; on the one hand between measured surface current and calculated depth-mean current; on the other hand between measured and estimated surface current with the exhibit method. The chosen point is situated in Mer d'Iroise in front of Brittany peninsula. The depth of water is about $150 \mathrm{~m}$ there and the average current $1 \mathrm{~m} / \mathrm{s}$, so the parameter $t$ is about $150 \mathrm{~s}$. You can note that the current at the surface is stronger than 


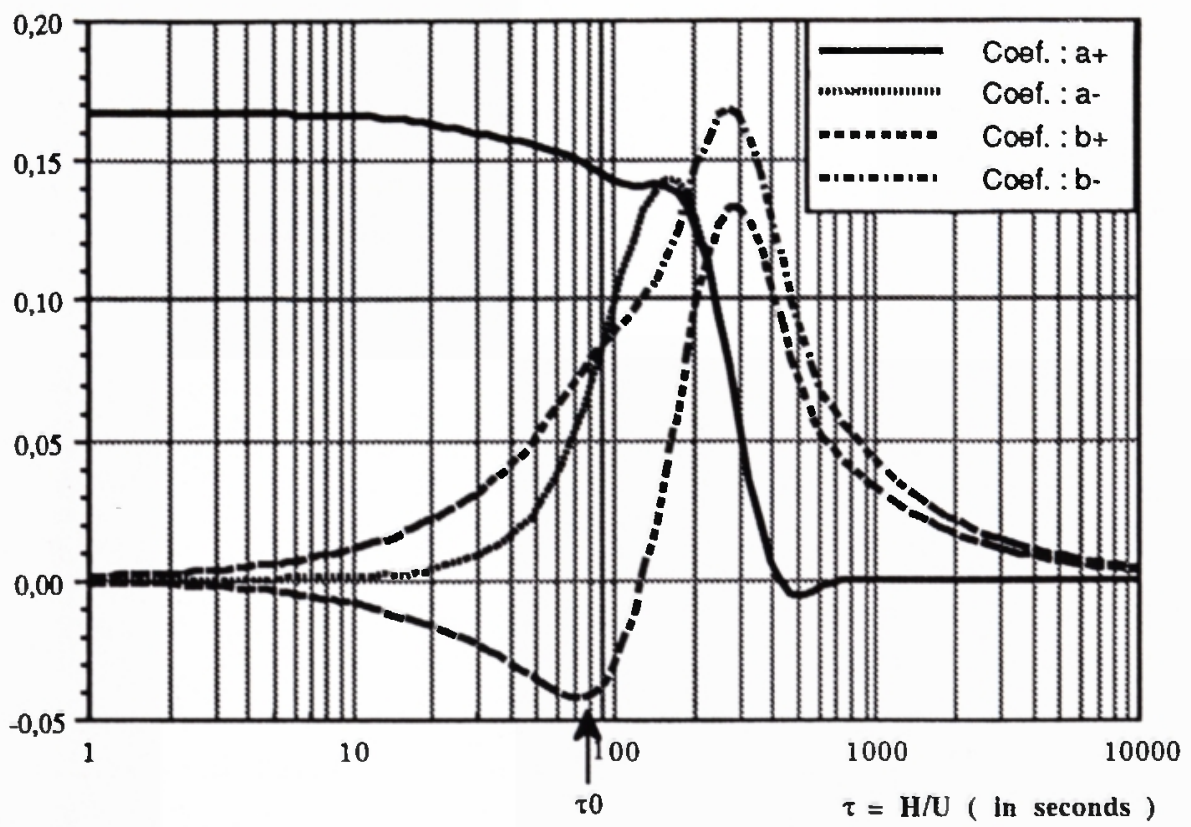

Fig. 1. - Coefficients of the matrix that transforms depth-mean current into surface current.
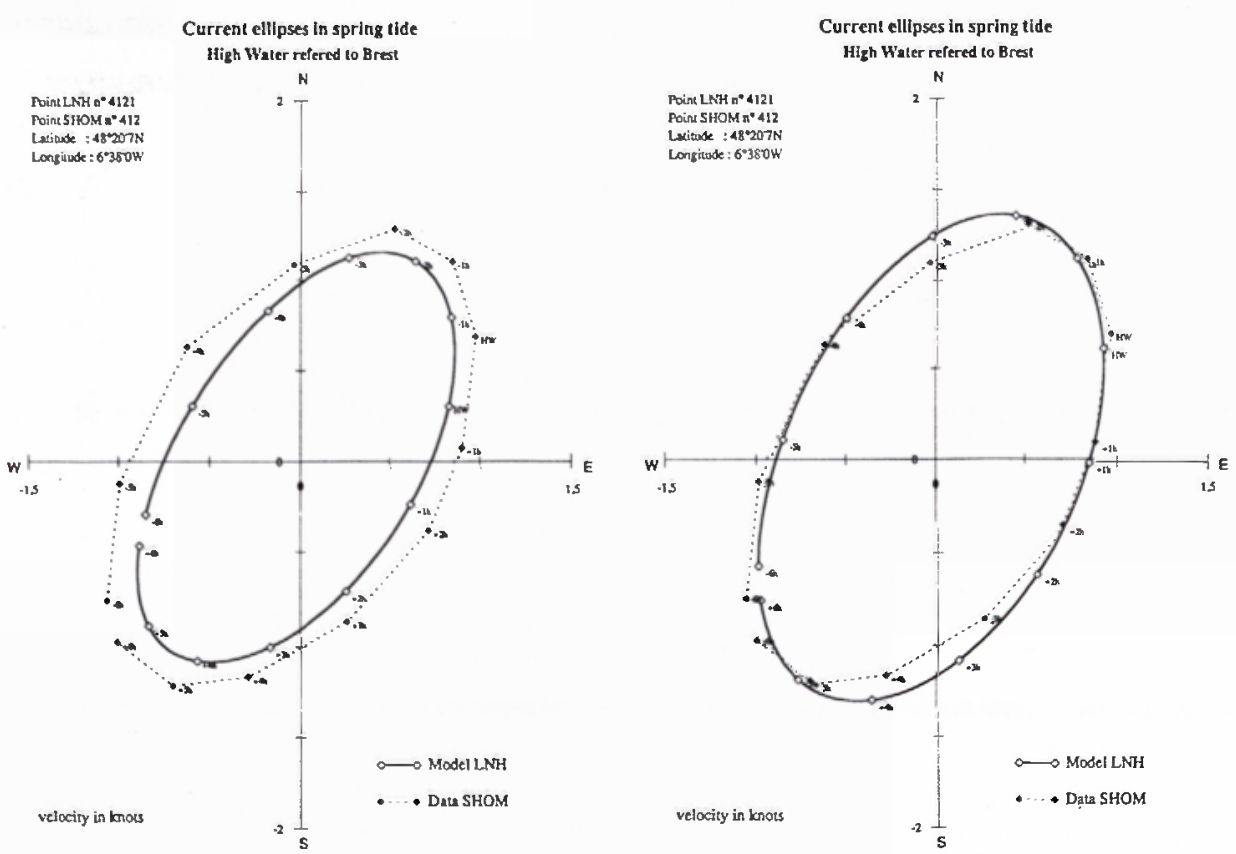

Fig. 2. - Effect of the transformation: on the left depth-mean current given by Telemac; on the right estimated surface current. 
the depth-mean current and also rotates with about an hour's delay; such a remark has already been exposed by Bowden [11].

\section{RESULTS AND COMPARISONS}

The results of simulations for each component regarding elevation have been successfully compared with maps made by Chabert d'Hières and Le Provost [4] and measures given by [5] and [6].

The amplitudes and phases of semi-diurnal waves of the model are in good agreement. Regarding the main component $\mathrm{M} 2$, the variations are lower than $20 \mathrm{~cm}$ for the amplitudes and 10 degrees for the phases all over the area.

The amplitude and phase of its harmonic M4 are in good agreement also, especially in the North Sea and in the eastern part of the English

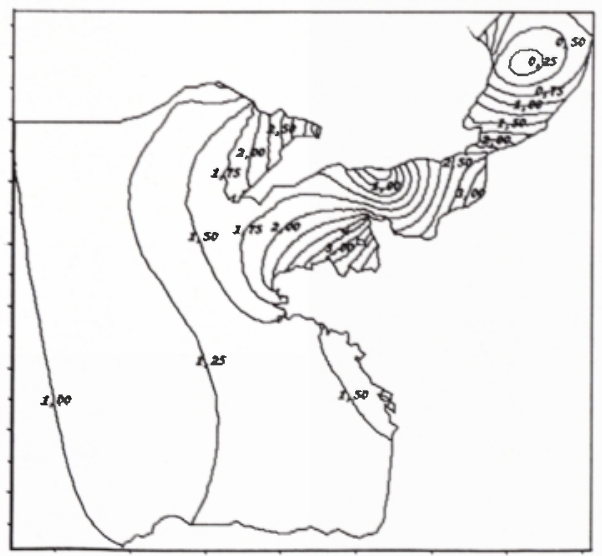

Channel where the two amphidromic points are correctly situated. More consistent bias appear in the Bay of Biscay and in the western part of the English Channel where the influence of the shelf edge is prevalent. Nevertheless, no amplitude bias is greater than $5 \mathrm{~cm}$ there.

Concerning the current components two kinds of comparaisons has been achieved.

The first one in the English Channel was made with the results obtained by Fornérino [1]. We compared directly the depth-mean currents components by means of a decomposition in 4 characteristic parameters : rent

- amplitude of the maximum cur-

- phase of the maximum current

- ratio of the minimum current to the maximum current

- direction of the maximum current

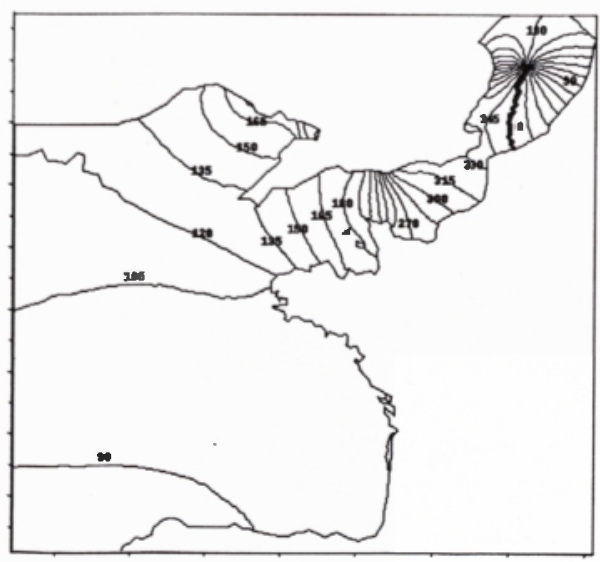

Fig. 3. - Semi-amplitude and phase of M2 wave elevation all over the domain. 

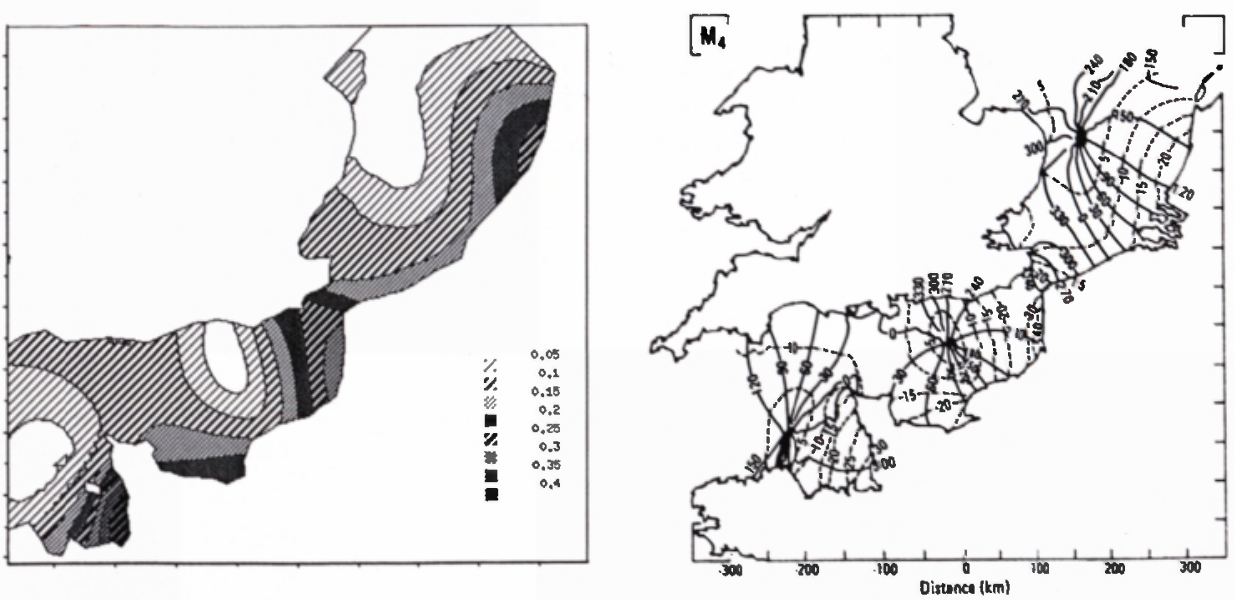

Fig. 4. - Semi-amplitude of M4 wave elevation in the English Chanel and the North Sea; on the lett LNH model; on the right Tidal Flow Forum.
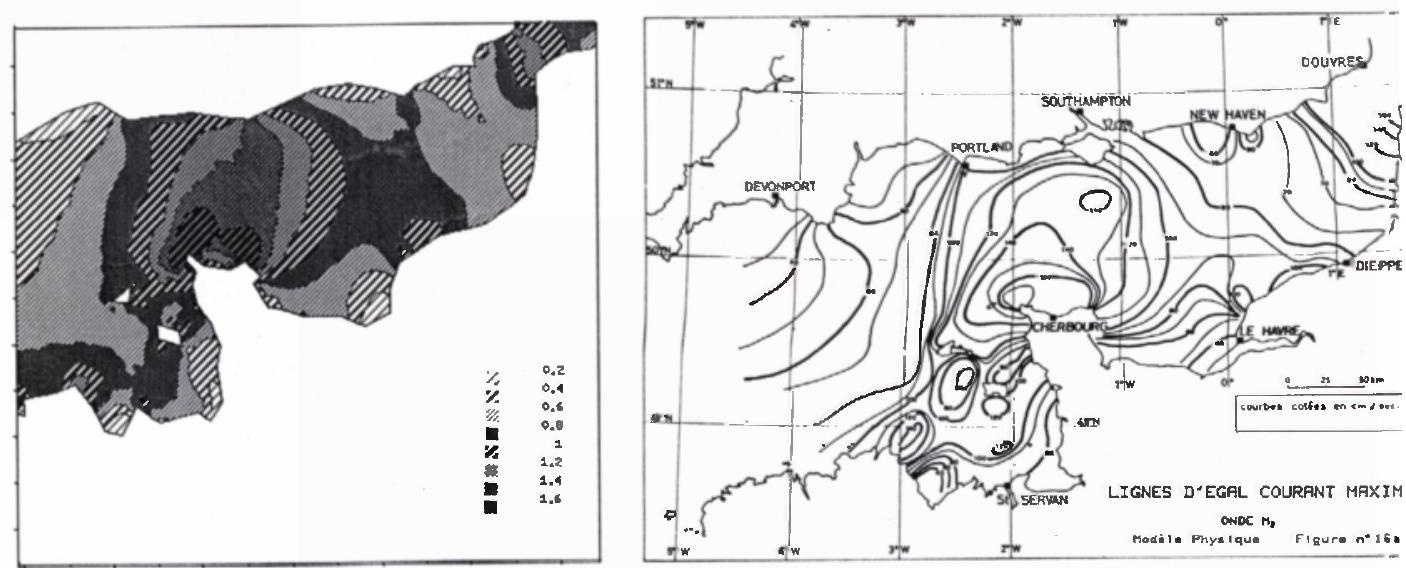

Fig. 5. - Amplitude of M2 maximum current in the English Chanel; on the left LNH model; on the right Fornérino's model

Qualitatively, no manifeste discordance came into sight. The place and the magnitude of the strongest currents fairly coincide and so do the positions of the amphidromic points (in that case, an amphidromic point is a point where the current vector describes a circle). However, it seems to be hard to go deeply into details as none of this models may be considered as a reference.

Another comparison has been realised with the measures made at the sea surface by the Hydrographic Service (SHOM) [14]. For each analysed point, we superposed the ellipse of 
the measured tidal mean spring current and the one obtained by the L.N.H. model and modified as described in the previous chapter. We consider that a mean spring current is obtained when M2 and S2 are perfectly in phase. Then we sum M2, S2 and M4 currents. We neglect N2 current as a mean spring tide does not inform about the phase relationship between this wave and the previous ones.

We got very good results in the Bay of Biscay and in the English Channel. At one and the same time, the sharpness of the ellipses, their axes and the phases coincided in these areas for more than $70 \%$ of points (see figure 2). The $30 \%$ of defective points are scattered all over the domain so we believe that it is due to local problems such as a bad interpolation of the bathymetry.

\section{CONCLUSIONS AND PROSPECTS}

The improvement in computer performances but also in numerical technics by which TELEMAC profits (non assembly of matrices, vectorizable matrix vector product...) are of great benefit for this tidal model, with an important reduction of computation efforts. Consequently, we could refine the mesh size wherever it was important to do it and the results that we obtained for elevation as well as for current components are in very good agreement with the measurements.
We now dispose of a complete data field on a monthly tidal cycle, with estimate vertical velocity profiles that we have already begun to use as boundary condition for local 3D models.

\section{ACKNOWLEDGEMENTS}

This study has been partially financed by the Service Technique de la Navigation Maritime et des Transmissions de l'Equipement within the framework of the E.D.F.-Ministries convention.

\section{REFERENCES}

[1] Fornerino, M., (1982), Modélisation des courants de marée dans la Manche, Thèse INPG, Grenoble, France.

[2] Galland, J.C., and Hervouet, J.M., (1990), Projet Saint-Venant - Code TELEMAC - version 1.0 - Note de principe, Rapport EDF HE-43/90.04, L.N.H., Chatou, France.

[3] Lepeintre, F., (1989), Comment résoudre les équations de Saint-Venant sphériques avecun code résolvant les équations de Saint-Venant cartésiennes - Application au code TELEMAC, Rapport EDF HE-41/89-28, L.N.H., Chatou, France.

[4] Chabert d'Hières, G., and Le Provost, C., (1978), Atlas des composantes harmoniques de la marée dans la Manche, Institut de Mécanique de Grenoble, France.

[5] Tidal Flow Forum, (1987), "6th International Conference on Computational Methods in Water Resources", Adv. in Water Resources, Vol. 10. 
[6] Cartwright, D.E., Edden, A.C., Spencer, R., and Vassie, J.M., (1980), The tides of the Northeast Atlantic ocean, Institute of Oceanographic Sciences, Bidston Observatory, Vol. 298, A. 1436, Birkenhead, Merseyside L43 7RA, U.K.

[7] Flather, R., (1976), "A tidal model of the north-west european continental shelf", Mémoires Société Royale des Sciences de Liège, 6e série, pp. 141-164.

[8] Galland, J.C., Janin, J.M., (1991), Simulation des courants de marée en Manche - Première partie, Rapport EDF HE-42/91.13, L.N.H., Chatou, France.

[9] Robinson, I.S., (1979), "The tidal dynamics of the Irish and Celtic Seas", Geophys. J. R. astr. Soc, Vol. 56, pp. 159-167.

[10] Davies, A.M., (1991), "On the accuracy of finite difference and modal methods for computing tidal and wind wave current profiles", international Journal for Numerical Methods in Fluids, Vol. 12, pp. 101-124.

[11] Bowden, K.F., (1983), Physical Oceanography of Coastal Waters, Ellis Horwood Ltd., Market Cross House, Chichester, West Sussex, PO19 1EB, U.K.

[12] Marchuk, G.I., Kagan, B.A., (1989), Dynamics of Ocean Tides, Kluwer Academic Publishers, P.O. Box 17, 3300 AA Dordrecht, The Netherlands.

[13] Schureman, P., (1971), Manual of Harmonic Analysis and Prediction of Tides, United States Government Printing Office, Washington D.C., U.S.A.

[14] SH No550, (1976), Courants de Marée dans la Manche et sur les Côtes Françaises de l'Atlatique, Service Hydrographique et Océanographique de la Marine, Paris, France. 